\title{
LiftYourself: A health promoting mobile application
}

\author{
Andreea Cojocaru \\ Department of Mathematics and \\ Computer Science \\ Ovidius University of Constanţa \\ Romania \\ andreea.cojocaru@365.univ- \\ ovidius.ro
}

\author{
Elena Băutu \\ Department of Mathematics and \\ Computer Science \\ Ovidius University of Constanța \\ Romania \\ ebautu@univ-ovidius.ro
}

\begin{abstract}
With the aim of promoting a healthy life with respect to physical activity and nutrition, this paper presents an attractive mobile application that engages the user on three directions. It proposes readymade fitness training sessions that target specific body areas, with the possibility to customize them as the user sees fit. The app allows the user to keep track of the nutrients consumed daily with respect to an ideal ratio that fits the individual profile of the user. Finally, menus are proposed to the user. Interactivity is supported by the incorporation of a speech based chatbot, developed using the IBM Watson technology. A questionnaire based usability study was conducted and measured the satisfaction level of the users that used the app, as well as gathered suggestions for improvement. This application has the potential to be extended for a wide range of uses, including clinical, preventive, public health or rehabilitation.
\end{abstract}

\section{Author Keywords}

Android; Mobile app; Health.

\section{ACM Classification Keywords}

H.5.2. Information interfaces and presentation (e.g., HCI): User Interfaces. H.3.2. Information Storage and Retrieval: Information Storage

\section{General Terms}

Human Factors; Design; Measurement.

DOI: $10.37789 /$ rochi.2021.1.1.18

\section{INTRODUCTION}

Stress is one the main triggers for depression, anxiety and sleep disorders. Both positive and negative experiences can lead to the appearance of some sensations of pressure or overwhelm. Lately, people are prone to a sedentary life, due to jobs in offices or inadequate nutrition, determined by the lack of motivation to prepare a healthy meal that takes time to prepare, in favor of an affordable fast food menu.
Many mobile fitness applications use the concept of gamification in order to motivate users to do more exercise [3]. Others have focused on competitiveness such as a ranking for community users, self-reflection as well as metaphorical approaches, such as visualization of garden flowers that grow according to each user's activity [4], or a combination of the two [1]. Many commercial fitness products, such as $\mathrm{Nike}^{6}$, Fitbit $^{7}$ and miCoach $^{8}$, adopt community-based competition. Recent studies have identified crucial evidence to support social interaction as a key element in motivating users to perform physical activities in the project. In particular, such social interaction between group members includes exchange of physical activities, cooperating and competing [2]. Moreover, the use of physical health monitoring applications can be extended towards helping patients with certain mental illnesses, by triggering alerts in critical situations, thus helping patients to receive help [6].

This paper introduces LiftYourself - an Android application that aims to support users towards maintaining a healthy lifestyle through the facilities it offers, such as: adding personalized training, calculation of nutrients and macronutrients needed for consumption one day, weight history, a user-managed food list, and a chatbot for user support.

The rest of the paper proceeds as follows. The next section provides a short summary of papers that tackle the topic of developing health related applications. Next, we delve into the presentation of the proposed app, by presenting first the general functional and non-functional requirements, followed by a short description of its software architecture. Next, the main functionalities of the app are presented. We provide a comparison between the proposed app and two of the most downloaded apps in the Play Store, with respect to the features they provide. Finally, the usability study performed to assess, in this phase, the usability characteristics of the app, is briefly presented.

\footnotetext{
${ }^{6} \mathrm{http}: / /$ nikeplus.nike.com/plus/
}

${ }^{8}$ http://micoach.adidas.com/ 


\section{RELATED WORK}

A summary of the essential characteristics of the applications in the healthcare field is presented in [8], while listing a number of widely used applications that monitor the health and fitness of patients. Most of the revised applications are focused on self-monitoring of health. The conclusion of this review clearly highlights the positive role that applications of fitness have for the health of their users. Moreover, in [6], the author proposes the idea that the use of physical health monitoring applications can be extended towards helping patients with certain mental illnesses. Thus, mobile applications could triggers alerts in critical situations, thus helping patients to receive help at times when they most need it.

Mobile applications in the field of healthcare are appreciated by the general public, and the merits to improve and help maintain good health have a strong impact on the individual level [10]. The possibility to integrate in the same application the types of food consumed, the amount and types of exercise, even regular medical tests help supervise health condition and to promptly initiate interventions for correcting behaviors that would lead to the destabilization of health [10]. "What gets measured, gets improved" said Peter Drucker, following the fight against obesity, (which was genetically transmitted from generation to generation in his family) using technology and exercise. He discovered at the age of 50 that what he considered to be the fate of the family is in fact reversible, so he decided to start the fight against the metabolic syndrome, by effectively exercising and giving up harmful foods to his body subsequently coming out victorious.

Many studies concentrate on the use of competitiveness or cooperation in fitness applications. In [4], some users have considered the competition attractive, while others considered it useless and incompatible with the revised app. They also found that cooperation did not improve significantly if team members were anonymous. In [5], results of a qualitative study found that users were motivated by the cooperative workouts as a group, while in terms of competition, users would prefer to compare themselves with themselves than to compete with others.

The paper [9] delivers a bibliometric analysis of the field of fitness applications, based on the analysis of 481 scientific articles, published between 2011 and 2019 and indexed in the Scopus and ISI Web of Science databases. The revised articles fall within the scope of Medicine, Informatics and Health. The authors of the analysis note that the field of research is one of great interest and that expects important developments in the future.

\footnotetext{
${ }^{9}$ LiftYourself homepage https://gitlab.com/Asryi
}

The current paper fits this interest and aims to present an application that brings together many features useful for gaining and maintaining an overall state of health.

\section{PROPOSED SYSTEM}

Functional and nonfunctional requirements for the system are briefly presented, followed by the architecture of the system and a presentation of the mobile application proposed.

\section{Functional requirements}

The functional requirements were analyzed together with an expert fitness trainer, after thoroughly reviewing several selected similar applications. The main features for the proposed system include offering customized fitness training and menus, as well as to keep track of the daily calories consumed by the user, with respect to his own fitness profile and total energy expenditure. All the fitness exercises are to be presented with videos as well as meaningful descriptions.

The interaction with the system is supposed to take place by means of a mobile application, supported by Android devices. Apart from an easy to use interface, the system should offer support for automatically chatting with users on health related issues.

\section{Non functional requirements}

The application should be user friendly and easy to use for average smartphone users, familiar with the Android environment. Since the application involves personal data, it only allows for secured access, by means of personal accounts for each user (see Figure 1). The data in the application should be easily scalable, thus allowing for easy addition of new exercises and food lists or menus.
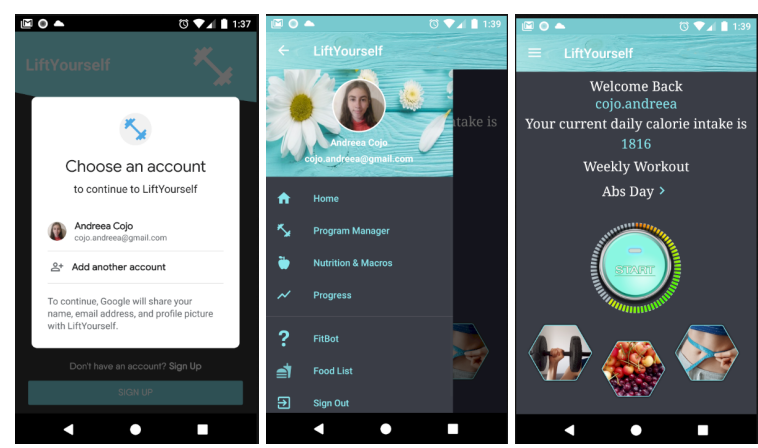

Figure 1. Main pages of the LiftYourself mobile app and the main menu.

\section{System components}

The LiftYourself mobile app is freely downloadable and ready to be tested ${ }^{9}$. The proposed system is implemented modularly, with highly cohesive, yet loosely coupled components that implement the functionalities (see Figure 
2). The data layer is at the foundation of the architecture, with data support offered both by a relational database, using SQLite, and by a NoSQL database, namely Firebase. The Android app is written in Java (minSdkVersion 21), being built for phones that run Android 8.0 (Oreo) or higher. Each component is implemented in one or more Java classes, such that the code is easily maintained and extended, if necessary.

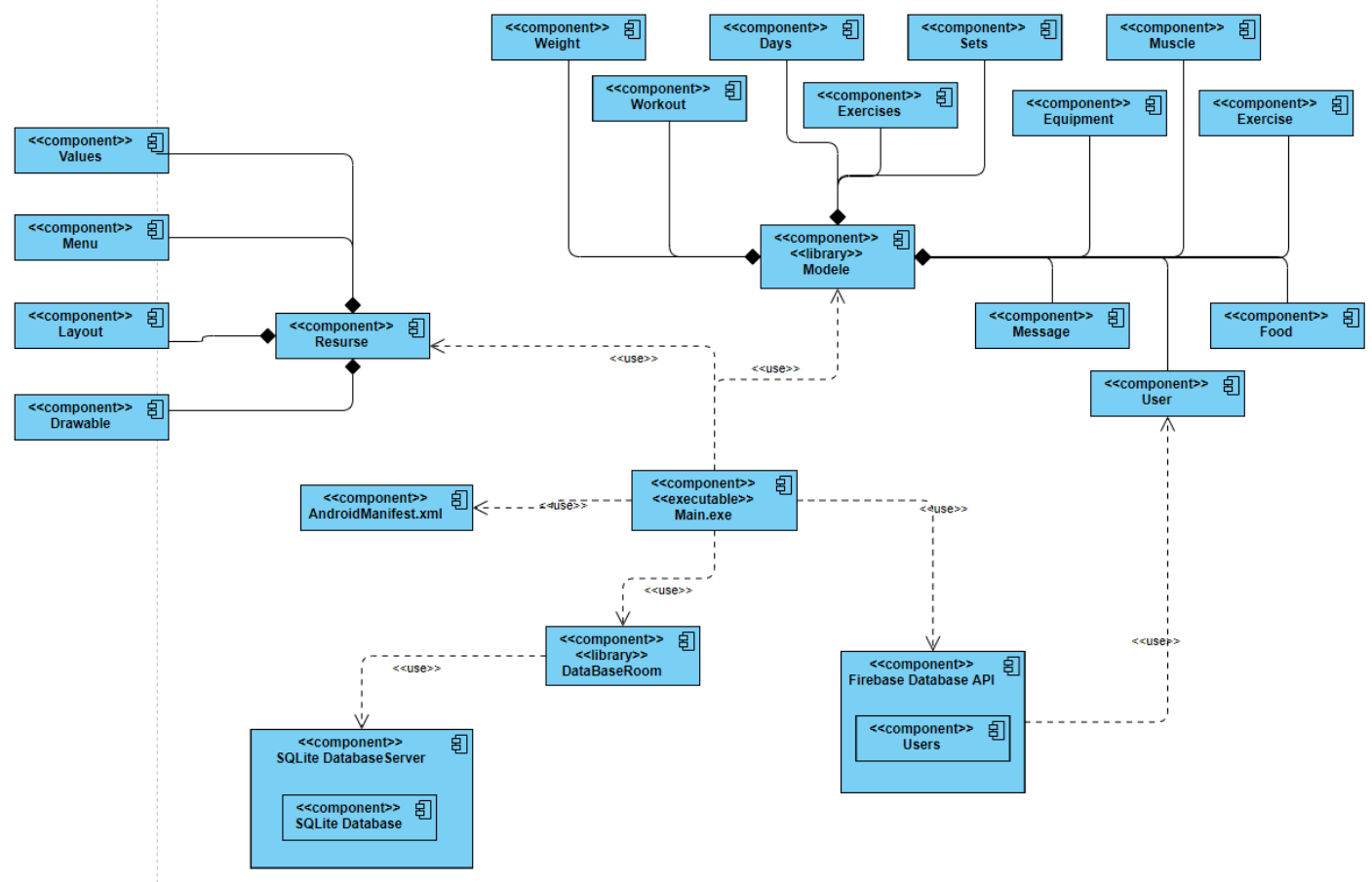

Figure 2: Component diagram of the system.

\section{FitBot}

Chatbots are software apps that can carry on an online conversation in either text mode, or text-to-speech mode, thus filling the place for a human dialogist [7]. Fitbot is the chatbot component implemented for the LiftYourself app. It uses the IBM Watson technology, using natural language processing and Speech-to-Text in order to provide fast and accurate transcription of spoken words in several languages for a variety of use cases. Examples of dialogues with Fitbot are presented in Figure 3.

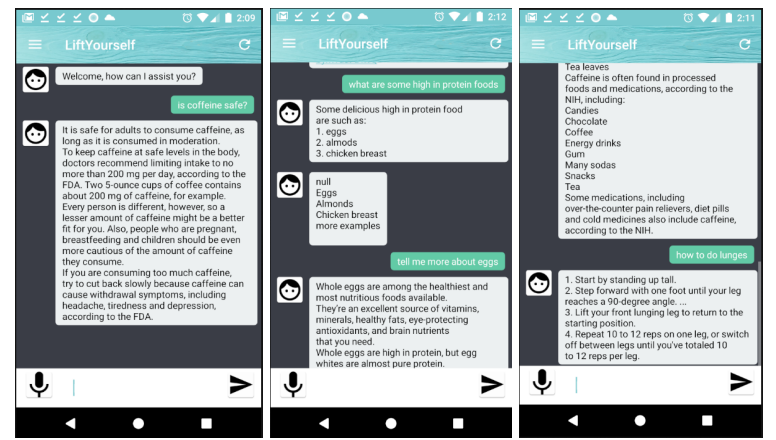

Figure 3. Example dialogues with Fitbot.

\section{Android App}

LiftYourself is the mobile app implemented to suit the functionalities mentioned previously. It only allows for authenticated users, either by logging in using an email address, either by their Google account (see Figure 1). The main window presents a summary of the calories the user is supposed to consume that day, according to the fitness plan previously chosen. Also, the main page allows easy access to the sections of the app: training sessions, nutrition and weight control. In the main window, by pressing the Start button, the user can start the predefined training session, while the device starts a mild vibration, in order to energize the user. The next page presents the list of fitness exercises that compose the current training session, with a video explaining it, indications with respect to the number of repetitions and a timer to keep track of the time between the exercises (see Figure 4). 

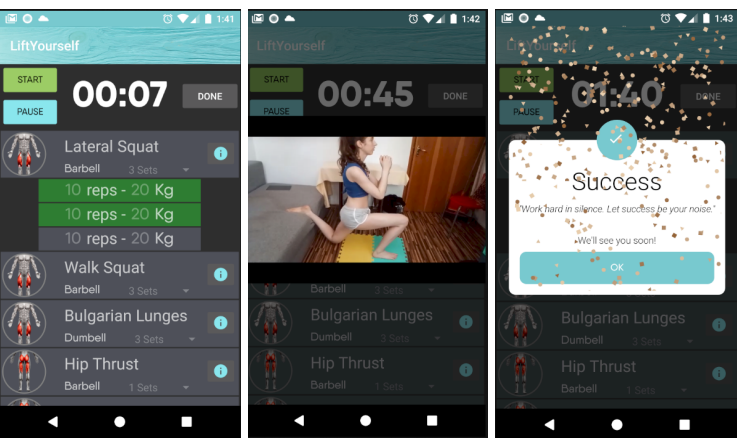

Figure 4. Pages for a chosen training.

Users can choose among some predefined training sessions and they can also propose new customized training sessions, since the app allows the introduction, update or delete of training sessions. Also, the user can tweak a given predefined training session, by changing the order of the exercises, or the number of repetitions, by adding or removing fitness exercises from the list that composes the session.
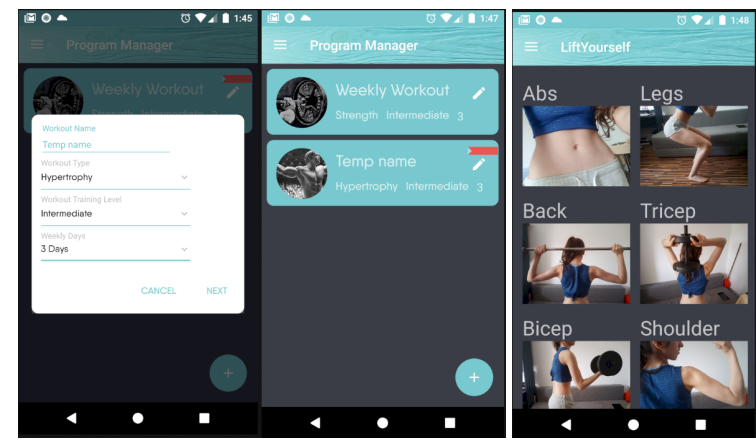

Figure 5. Pages for a chosen training.

When first accessing the Nutrition and Macros page, the user must define his profile, by providing information related to his physical appearance and fitness habits. The app computes the total daily energy expenditure of the user and provides an approximate number of calories for the user, depending on his fitness goal (see Figure 6). The app keeps track of the evolution of the user's weight and presents it by using line charts.
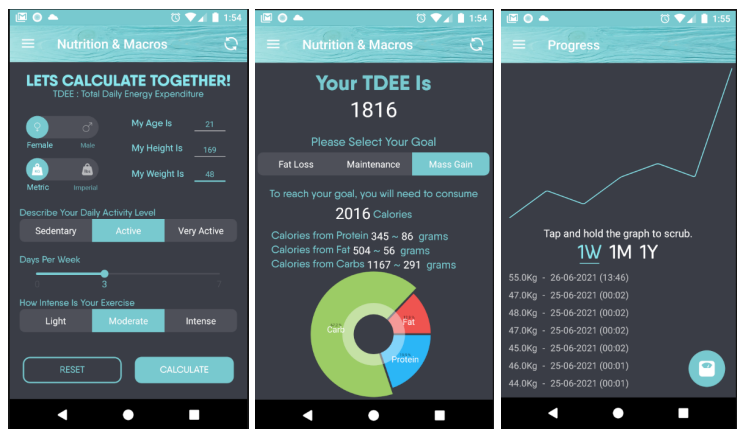

Figure 6. Nutrition and Macros and progress visualization.

Finally, the third section of the app is the food list, where the user can record the food eaten, uploading a picture and information on calories and nutrients contained, in order to keep track of calories consumed (Figure 7).
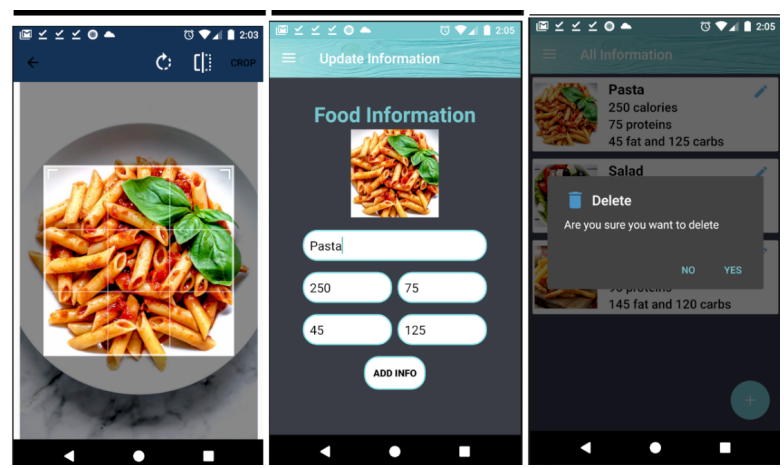

Figure 7. Food list and calorie tracking.

\section{SIMILAR APPLICATIONS}

HomeWorkout ${ }^{10}$ is the most popular app in Play Store, with over 100 million downloads. It presents a multitude of readily defined training sessions, named challenges, that target specific muscle groups or body areas. Each exercise has a brief description and demonstrative video.

\footnotetext{
${ }^{10}$ Home workout homepage

https://play.google.com/store/apps/details?id=homeworkout .homeworkouts.noequipmenthl=engl=US
} 


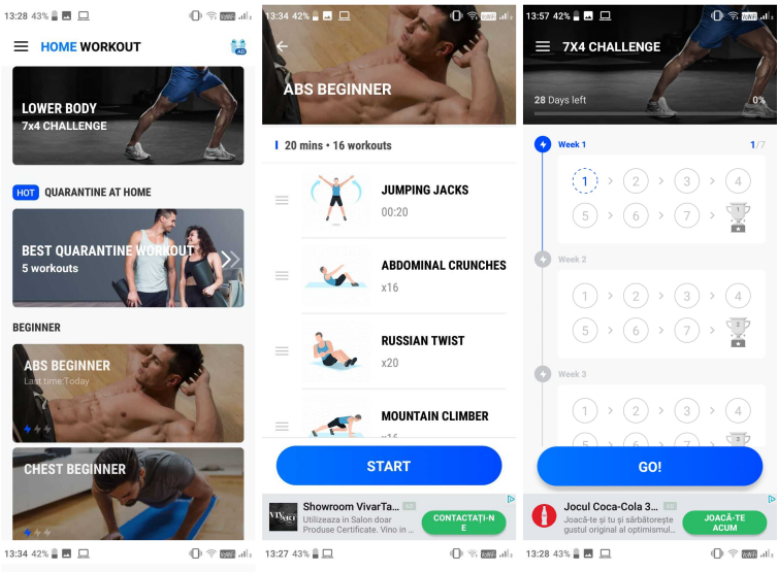

Figure 8. Pages for a chosen training.

FitOn ${ }^{11}$ is another popular app in the PlayStore, which focuses on offering training sessions as well as nutrition, by providing users with personalized menus. It also involves a social part, by the creation of friend lists with which the user can chat and motivate each other.

Table 1 presents a comparison of the proposed application, LiftYourself, with the two popular application mentioned above, with respect to the features they offer.

\begin{tabular}{|c|c|c|c|}
\hline Feature & $\begin{array}{c}\text { Lift } \\
\text { Yourself }\end{array}$ & $\begin{array}{c}\text { Home } \\
\text { workout }\end{array}$ & FitOn \\
\hline $\begin{array}{c}\text { visualization } \\
\text { physical } \\
\text { exercises }\end{array}$ & Yes & Yes & Yes \\
\hline food recipes & No & No & Yes \\
\hline $\begin{array}{c}\text { custom food } \\
\text { list }\end{array}$ & Yes & No & No \\
\hline $\begin{array}{c}\text { personalized } \\
\text { physical } \\
\text { exercises }\end{array}$ & Yes & No & No \\
\hline friends list & No & No & Yes \\
\hline $\begin{array}{c}\text { evidence of } \\
\text { progress }\end{array}$ & Yes & Yes & No \\
\hline $\begin{array}{c}\text { chatbot / } \\
\text { assistant / } \\
\text { coach }\end{array}$ & Yes & No & Yes \\
\hline $\begin{array}{c}\text { interesting } \\
\text { articles }\end{array}$ & No & No & Yes \\
\hline $\begin{array}{c}\text { evidence of } \\
\text { food calories }\end{array}$ & Yes & No & Yes \\
\hline
\end{tabular}

${ }^{11}$ FitOn homepage https://fitonapp.com/

\begin{tabular}{|c|c|c|c|}
\hline $\begin{array}{c}\text { evidence of } \\
\text { calories burned }\end{array}$ & No & Yes & Yes \\
\hline $\begin{array}{c}\text { intuitive } \\
\text { interface }\end{array}$ & Yes & Yes & Yes \\
\hline $\begin{array}{c}\text { nutrient } \\
\text { computer }\end{array}$ & Yes & No & No \\
\hline
\end{tabular}

Table 9. Comparison between similar applications.

\section{USABILITY TESTING}

The usability of the application was assessed by means of a small study that involved 13 anonymous real users that have used the app, in the 20 to 30 years old age group. The study presents merely a preliminary usability evaluation, since the size of the sample group is not sufficient in order to provide a comprehensive usability evaluation. The users weren't instructed on how to use the app, being left to discover by themselves its features. After using the app, they filled in a questionnaire where questions were to be answered on a Likert 10 points scale.

Most of the users found the app very easy to use, with only 3 users having considered the app as hard to use. Depending on the physical training of each user, the result differs. People who already practice regularly, found it easier to follow the exercises in the application.

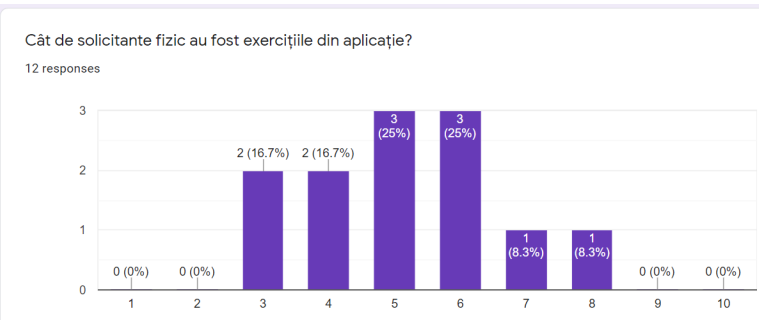

Figure 9. Distribution of answers to the question "How physically demanding were the exercises in the application?"

The degree to which the users were satisfied with the results after using the application is depicted graphically in Figure 10 We should also take into account the subjectiveness of the answers to this question, because the answers depend on the degree of involvement and commitment of the users to physical training. 


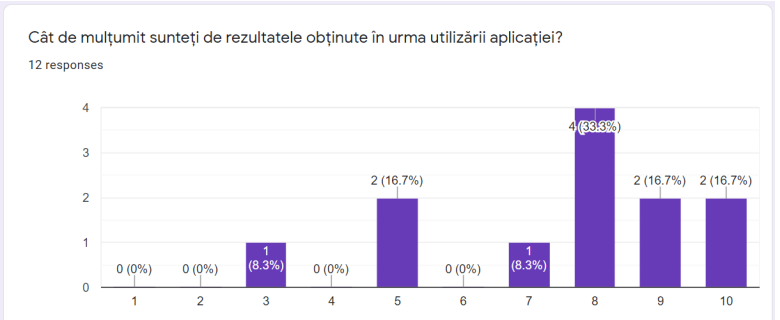

Figure 10. Distribution of answers to the question "How satisfied you are with the results of the use application?"

Committed users didn't find it very hard to get the results they have hoped for (Figure 10).

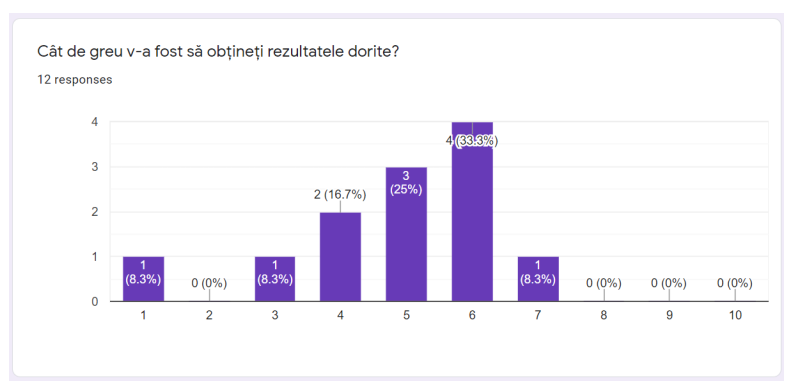

Figure 11. Distribution of answers to the question "How hard was it for you to get the results you wanted?"

The app seems to have helped the majority of its users with respect to achieving health related goals, since more than $90 \%$ of the users answered Yes to question no. 7 (see Figure 12. Also, the vast majority of users agree that the app helps them live healthier.

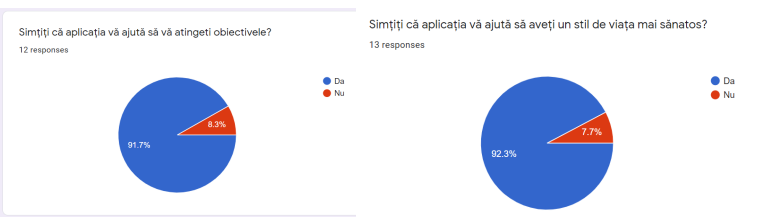

Figure 12. Distribution of answers to the questions (left) "Do you feel that the application helps you achieve your goals?", (right) "Do you feel that the application helps you achieve a healthier lifestyle?"

Finally, users were asked to provide suggestions for improvement. While $85 \%$ of the users didn't have any suggestions, one user suggested the removal of the vibration at the start of training sessions, and also specified that he didn't understand the point of the Progress button, while another user suggested to incorporate fitness exercises other that calisthenics and to provide more meal plans.

\section{CONCLUSION}

This paper presented in brief a mobile application meant to help its users achieve a healthier lifestyle. The proposed system brings together features commonly exhibited by fitness training apps, as well as the possibility to track users' menu and compute various calorie calculations.

\section{ACKNOWLEDGMENTS}

We thank all the volunteers that tested the app and provided valuable feedback.

\section{REFERENCES}

1. Chen, Y., \& Pu, P. (2014, April). HealthyTogether: exploring social incentives for mobile fitness applications. In Proceedings of the second international symposium of chinese chi (pp. 25-34).

2. Chen, Y., Chen, Y., Randriambelonoro, M. M., Geissbuhler, A., \& Pu, P. (2017, February). Design considerations for social fitness applications: comparing chronically ill patients and healthy adults. In Proceedings of the 2017 ACM Conference on Computer Supported Cooperative Work and Social Computing (pp. 1753-1762).

3. Tu, R., Hsieh, P., \& Feng, W. (2019). Walking for fun or for "likes"? The impacts of different gamification orientations of fitness apps on consumers' physical activities. Sport Management Review, 22(5), 682-693.

4. Lin, J. J., Mamykina, L., Lindtner, S., Delajoux, G., \& Strub, H. B. (2006, September). Fish'n'Steps: Encouraging physical activity with an interactive computer game. In International conference on ubiquitous computing (pp. 261-278). Springer, Berlin, Heidelberg.

5. Ahtinen, A., Isomursu, M., Mukhtar, M., Mäntyjärvi, J., Häkkilä, J., \& Blom, J. (2009, November). Designing social features for mobile and ubiquitous wellness applications. In Proceedings of the 8th international Conference on Mobile and Ubiquitous Multimedia (pp. 1-10).

6. Arthur, F., Siegel, J.. Suicide prevention by smartphone. The American Journal of Medicine, 129(8):e145, 2016

7. Khan, R., \& Das, A. (2018). Build better chatbots. A complete guide to getting started with chatbots, ISBN ISBN: 978-1-4842-3111-1.Springer.

8. Higgins, J.P. Smartphone applications for patients' health and fitness. The American Journal of Medicine, 129(1):11-19, 2016.

9. Liu, Y. and Avello, M.. Status of the research in fitness apps: A bibliometric analysis. Telematics and Informatics, 57:101506, 2021.

10. Powers Hannley, P. The 'Springer gene': Combating familial obesity with technology and exercise. The American Journal of Medicine, 129(1):5-7, 2016. 